\title{
Calibration method for multiangle lidar measurements
}

\author{
Markus Pahlow, Vladimir A. Kovalev, and Marc B. Parlange
}

\begin{abstract}
A new method based on a two-angle approach is developed to determine the lidar solution constant from scanning elastic lidar data, hence providing a relative calibration for each lidar scan. Once the solution constant is determined, the vertical profiles of atmospheric extinction can be calculated. With this calibration method a minimization technique is used that replaces the linear regression used in a known two-angle approach that requires only local atmospheric homogeneity over a restricted altitude calibration range rather than overall horizontal homogeneity. Lidar signals from at least one pair of elevation angles are used, averaged in time when the system is operated in a permanent two-angle mode, or an arbitrary number of signal pairs is used, when a two-dimensional lidar scan is being processed. The method is tested extensively with synthetic data. The calibration method is a robust tool for determining the solution constant to the lidar equation and for obtaining vertical profiles of atmospheric extinction. (C) 2004 Optical Society of America
\end{abstract}

OCIS codes: $280.3640,280.1100,290.1310$.

\section{Introduction}

In the general case of a two-component atmosphere the lidar return signal $P(r)$ is related to atmospheric backscatter and extinction in the following way:

$$
\begin{aligned}
P(r)= & C_{0} E T_{1}{ }^{2} \frac{\beta_{p}(r)+\beta_{m}(r)}{r^{2}} \exp \left\{-2 \int_{r_{1}}^{r}\left[\kappa_{p}(x)\right.\right. \\
& \left.\left.+\kappa_{m}(x)\right] \mathrm{d} x\right\},
\end{aligned}
$$

where the background constituent $P_{b g r}$ has been subtracted. Here $C_{0}$ is a lidar system constant, $E$ is the energy of the emitted light pulse, and $T_{1}^{2}$ is the twoway transmittance over the range from $r=0$ to $r_{1}$, where $r_{1}$ is the minimum lidar measurement range, generally the first range gate of the zone of complete overlap between the field of view of the telescope and the laser beam. The particulate and molecular

\footnotetext{
When this work was done M. Pahlow and M. B. Parlange were with the Department of Geography and Environmental Engineering, Johns Hopkins University, Baltimore, Maryland 21218. V. A. Kovalev is with the Fire Sciences Laboratory, U.S. Department of Agriculture, P.O. Box 8089, Missoula, Montana 59807. M. Pahlow is now at the Environmental Technology Laboratory, National Oceanic and Atmospheric Administration, Boulder, Colorado 80305 (e-mail, markus.pahlow@noaa.gov).

Received 4 August 2003; revised manuscript received 23 December 2003; accepted 9 February 2004.

0003-6935/04/142948-09\$15.00/0

(C) 2004 Optical Society of America
}

backscatter and extinction coefficients are denoted as $\beta_{p}, \beta_{m}, \kappa_{p}$, and $\kappa_{m}$, respectively.

Indeterminacy of the lidar equation [Eq. (1)] leads to additional requirements in order for a solution to the inversion problem to be determined. One possible approach is two-angle or multiangle methods. Development of two-angle or multiangle methods to invert the lidar equation was initiated in the classic work described in Refs. 1 and 2. To date there are several approaches to the problem. Owing to the indeterminacy of the lidar equation, any inversion algorithm has to resort to certain assumptions in order for one to solve for atmospheric backscatter and extinction. Commonly multiangle inversion methods either are based on an assumption of horizontally homogeneous atmosphere or employ reference or $a$ priori data. Moreover conventional multiangle inversion methods assume that either the effective vertical transmission is homogeneous for all slant paths through the layer; i.e., it is uniquely related to the elevation angle ${ }^{3-5}$ or that scattering within thin horizontal layers is also homogeneous. ${ }^{1,2}$ Sasano and Nakane ${ }^{6}$ proposed a multiangle method that was developed specifically for high-altitude measurements of the troposphere and assumes an aerosol-free zone within the lidar measurement range. With this method the boundary conditions are estimated through an iterative procedure, and the procedure is carried out until the solution converges. This method has been applied successfully to derive aerosol optical properties from lidar data of the troposphere to as high as an altitude of $12 \mathrm{~km}$, where the 
assumption of an aerosol-free area was applicable, at least before the Mt. Pinatubo eruption took place. ${ }^{7,8}$

Another approach to multiangle lidar measurements was developed by Gutkowicz-Krusin. ${ }^{9}$ In this work the assumption of a unique relationship between optical depth and elevation angle is also invoked. Model calculations are presented that show that, in addition to being applicable in horizontally homogeneous atmosphere, the method can determine the overall transmission of optically thin inhomogeneities, also in the presence of shot noise. More recently a variational method for determining aerosol optical thickness and backscatter coefficient profiles from multiangle lidar measurements has been devised. ${ }^{10}$ In this case a horizontally homogeneous atmosphere and a unique relationship between the elevation angle and the optical depth of the layer under investigation are assumed. The method was applied successfully to a data set obtained during the INDOEX campaign, as shown by comparison with simultaneous sunphotometer measurements.

A significant drawback of the conventional algorithms for multiangle lidar measurements is that they do not allow for use of relevant spatial or time averaging of either original or transformed lidar data, which would significantly reduce the influence of local inhomogeneities and signal noise. The two-angle solutions described in Refs. 11 and 12 allow one to apply averaging either in time or in space. Furthermore horizontal atmospheric homogeneity is required only locally and in properly selected angular sectors and altitude ranges. We present a method for determining the lidar solution constant for a two-component atmosphere, based on these two-angle approaches. The method requires having the signal profiles of lidar measurements at least at two elevation angles to determine the lidar solution constant, i.e., to perform a relative calibration. The vertical profile of atmospheric extinction can then be derived, once the lidar solution constant is known.

\section{General Lidar Equation Solution for Two-Angle or Multiangle Measurements}

First we investigate the general lidar equation solution in the context of two-angle or multiangle measurements. It is helpful to introduce the two-angle method (TAM), which was developed for single-ended multiangle lidar measurements. ${ }^{11,13}$ The concept behind the method is as follows. In the general case of multiangle measurements the lidar scans the atmosphere in many angular directions at a constant azimuth, starting from a direction close to horizontal (also known as a range-height indicator scan). In order to determine the lidar solution constants, lidar signals $P_{1}(r)$ and $P_{2}(r)$, measured at elevation angles $\varphi_{1}$ and $\varphi_{2}$, are selected (Fig. 1). Let us begin the method development by range correcting and transforming the background subtracted lidar signals $P_{1}(r)$ and $P_{2}(r)$ before inversion is made. With auxiliary correction functions $Y_{1}(r)$ and $Y_{2}(r)^{14}$ the range-

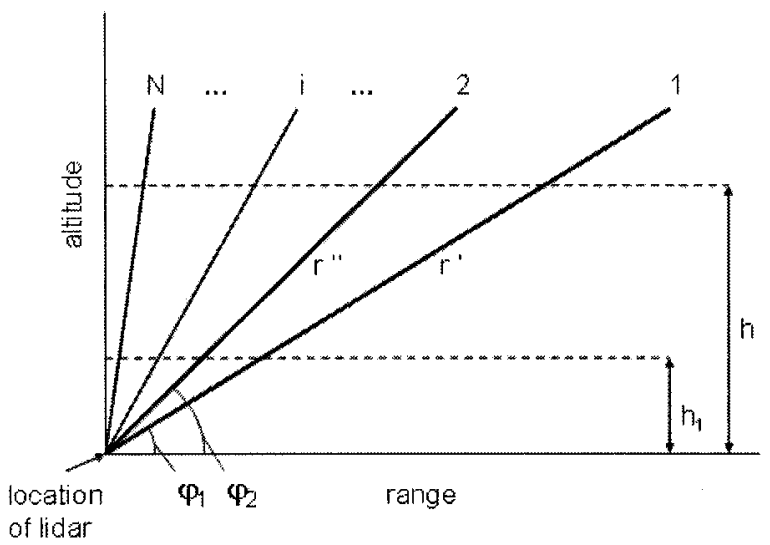

Fig. 1. Schematic of TAM.

corrected lidar signal at altitude $h$ and elevation angles $\varphi_{1}$ and $\varphi_{2}$ can be transformed to

$$
\begin{aligned}
& S_{1}(h)=P_{1}\left(r^{\prime}\right) Y_{1}\left(r^{\prime}\right) r^{\prime 2}, \\
& S_{2}(h)=P_{2}\left(r^{\prime \prime}\right) Y_{2}\left(r^{\prime \prime}\right) r^{\prime \prime 2},
\end{aligned}
$$

where $r^{\prime}=h / \sin \varphi_{1}, r^{\prime \prime}=h / \sin \varphi_{2}$,

$$
\begin{aligned}
& Y_{1}\left(r^{\prime}\right)=C_{Y} \Pi_{p}\left(r^{\prime}\right)^{-1} \exp \left\{-2 \int_{r_{1}^{\prime}}^{r^{\prime}}[a(x)-1] \kappa_{m}(x) \mathrm{d} x\right\}, \\
& Y_{2}\left(r^{\prime \prime}\right)=C_{Y} \Pi_{p}\left(r^{\prime \prime}\right)^{-1} \exp \left\{-2 \int_{r_{1^{\prime \prime}}}^{r^{\prime \prime}}[a(x)-1] \kappa_{m}(x) \mathrm{d} x\right\} .
\end{aligned}
$$

The constant $C_{Y}$ is arbitrary, and $a(r)=\Pi_{m}(r) / \Pi_{p}(r)$ denotes the ratio of the molecular to particulate backscatter-to-extinction ratio. If no molecular absorption occurs at the wavelength of the emitted light pulse, then $\Pi_{m}=3 /(8 \pi)$. The range of typical particulate backscatter-to-extinction ratios $\Pi_{p}$ is $\sim 0.01$ $0.05 \mathrm{sr}^{-1} .7,15-19$

The weighted extinction coefficient for elevation angles $\varphi_{1}$ and $\varphi_{2}$, can be written, respectively, as

$$
\begin{aligned}
& \kappa_{W, 1}(h)=\frac{S_{1}(h)}{C_{1}-2 I_{1}\left(h_{1}, h\right)}, \\
& \kappa_{W, 2}(h)=\frac{S_{2}(h)}{C_{2}-2 I_{2}\left(h_{1}, h\right)},
\end{aligned}
$$

where $C_{1}$ and $C_{2}$ are the lidar equation constants and $h_{1}$ is the selected minimum altitude of the atmospheric layer under investigation. Integrals $I_{1}\left(h_{1}\right.$, $h)$ and $I_{2}\left(h_{1}, h\right)$ in Eqs. (4) and (5) are

$$
\begin{aligned}
& I_{1}\left(h_{1}, h\right)=\int_{h_{1} / \sin \varphi_{1}}^{h / \sin \varphi_{1}} S_{1}(x) \mathrm{d} x, \\
& I_{2}\left(h_{1}, h\right)=\int_{h_{1} / \sin \varphi_{2}}^{h / \sin \varphi_{2}} S_{2}(x) \mathrm{d} x,
\end{aligned}
$$

respectively.

Assuming a range-independent backscatter-to- 
extinction ratio, the weighted extinction coefficient $\kappa_{W}(h)$ in Eqs. (4) and (5) is defined as

$$
\kappa_{W}(h)=\kappa_{p}(h)+a \kappa_{m}(h) .
$$

To estimate the influence of atmospheric inhomogeneity, one should derive and analyze the solution to the two-angle lidar equation in its most general form. Such a general solution can be obtained from Eqs. (4) and (5), assuming that $\kappa_{W, 1}(h) \neq \kappa_{W, 2}(h)$. The general TAM equation can then be expressed as

$$
\frac{S_{1}(h)}{S_{2}(h)}=\frac{C_{1} \kappa_{W, 1}(h)}{C_{2} \kappa_{W, 2}(h)}\left[\frac{1-2 I_{1}\left(h_{1}, h\right) / C_{1}}{1-2 I_{2}\left(h_{1}, h\right) / C_{2}}\right] .
$$

The solution may be written in the form

$$
\begin{aligned}
y(h)= & \frac{1}{1-\left[1-\frac{\kappa_{W, 2}(h)}{\kappa_{W, 1}(h)}\right] \exp \left[-2 \int_{h_{\min }}^{h} \kappa_{W, 2}\left(h^{\prime}\right) \mathrm{d} h^{\prime}\right]} \\
& \times\left[\frac{C_{1}}{C_{2}}-\frac{2}{C_{2}} x(h)\right],
\end{aligned}
$$

with $x(h)=S_{1}(h) / S_{2}(h)$ and $y(h)=2 I_{1}\left(h_{1}, h\right)-$ $2 I_{2}\left(h_{1}, h\right) S_{1}(h) / S_{2}(h)$. As follows from Eq. (10) the sensitivity of the solution to horizontal inhomogeneity depends on the ratio of $\kappa_{W, 2}(h)$ to $\kappa_{W, 1}(h)$ and on the level of atmospheric attenuation. For clear atmospheres and restricted altitude ranges $\left(h_{\min }, h\right)$ the second term in the denominator on the right-hand side of Eq. (10) is

$$
\exp \left[-2 \int_{h_{\min }}^{h} \kappa_{W, 2}\left(h^{\prime}\right) \mathrm{d} h^{\prime}\right] \approx 1 ;
$$

hence

$$
y(h) \approx \frac{\kappa_{W, 1}(h)}{\kappa_{W, 2}(h)}\left[\frac{C_{1}}{C_{2}}-\frac{2}{C_{2}} x(h)\right] .
$$

This solution is asymmetric and discussed in more detail below. For turbid atmospheres the influence of the horizontal inhomogeneity decreases as the optical depth of the layer $\left(h_{\min }, h\right)$ increases. Indeed in this case

$$
\exp \left[-2 \int_{h_{\min }}^{h} \kappa_{W, 2}\left(h^{\prime}\right) \mathrm{d} h^{\prime}\right] \ll 1,
$$

so that for a moderate and random difference between $\kappa_{W, 1}(h)$ and $\kappa_{W, 2}(h)$ the absolute value of the product of two terms in the denominator of Eq. (10),

$$
\left[1-\frac{\kappa_{W, 2}(h)}{\kappa_{W, 1}(h)}\right] \exp \left[-2 \int_{h_{\min }}^{h} \kappa_{W, 2}\left(h^{\prime}\right) \mathrm{d} h^{\prime}\right],
$$

decreases with the increasing optical depth of the layer. Accordingly Eq. (10) reduces to

$$
y(h) \approx \frac{C_{1}}{C_{2}}-\frac{2}{C_{2}} x(h),
$$

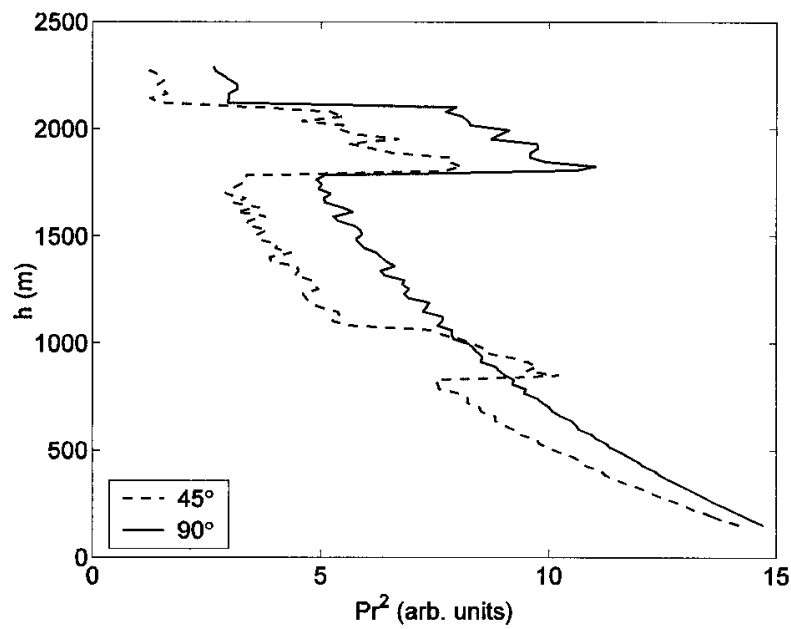

Fig. 2. Range-corrected lidar signals at a $45^{\circ}$ and a $90^{\circ}$ elevation angle for a TAM test.

where, unlike Eq. (12), the function $y(h)$ is not directly proportional to the ratio $\kappa_{W, 1}(h)$ to $\kappa_{W, 2}(h)$. This approximated solution in (15) is equivalent to the solution proposed in Ref. 11 for single-component polluted atmospheres, assuming horizontal homogeneity. Indeed, the approximated solution may be used in a turbid atmosphere, as in this case, nonuniformity of the particulate scattering within horizontal layers is less influential than in clear atmospheres [Eq. (12)].

To further clarify the two-angle concept and to evaluate the performance of the TAM, we use a synthetic data set as a test case. It is assumed that the model atmosphere under investigation is composed of molecules and particles. An aerosol plume located at $\sim 900 \mathrm{~m}$ and a cloud layer at $\sim 1900 \mathrm{~m}$ are the predominant structures in this model atmosphere. The corresponding lidar signals are shown in Fig. 2. The signal at a $45^{\circ}$ elevation angle has a mildly turbid layer from 850 to $1050 \mathrm{~m}$, corresponding to the local aerosol plume. At 1800-2100 m both signals increase owing to the strong turbidity caused by the cloud layer. Note that random noise has been added to the signals to represent typical lidar measurement noise. Also, these fluctuations resemble natural small-scale spatial horizontal heterogeneity in the particulate extinction coefficient profile. After the signals are transformed according to Eqs. (2) and (3), the integrals of the signals [Eqs. (6) and (7)] are computed. Then functions $x(h)\left[=S_{1}(h) / S_{2}(h)\right]$ and $y(h)\left[=2 I_{1}\left(h_{1}, h\right)-2 I_{2}\left(h_{1}, h\right) S_{1}(h) / S_{2}(h)\right]$ can be obtained. A crucial step in the TAM analysis, especially for data taken in mildly turbid or nonhomogeneous atmospheres, is the determination of the optimum range for regression. In the TAM developed by Ignatenk $0^{11}$ the entire range of variable pairs $[x(h), y(h)]$ was used to obtain coefficients $C_{1}$ and $C_{2}$ from linear regression. The methodology applied here differs from this approach. To determine the optimum values for lidar equation constants $C_{1}$ and $C_{2}$, the total regression range is divided into $n$ 


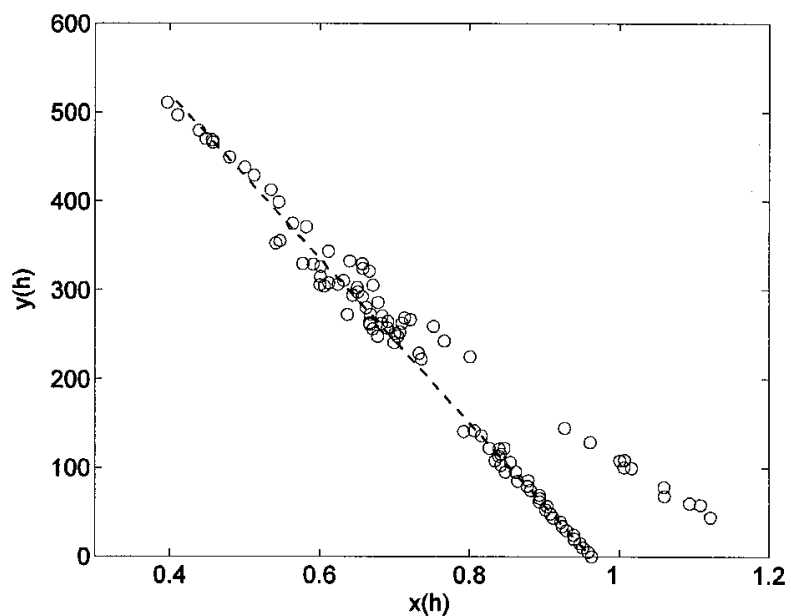

Fig. 3. Pairs of $[x(h), y(h)]$ for the test analysis. Also shown is the optimum regression line as obtained from the analysis.

subintervals. The total number of intervals must be chosen such that each subinterval contains a sufficient number of data points in order for reliable statistics to be obtained. The least-squares analysis is then performed for each individual interval, yielding $n$ pairs of lidar equation constants $C_{1}$ and $C_{2}$. To determine the optimum range, and at the same time the regression constants that give the best fit, we compute a set of five parameters for each interval. The parameter set consists of the standard deviation of $C_{1}, \sigma_{C 1}$, the normalized standard deviation of $C_{1}$, $\sigma_{C 1, \text { norm }}$, the standard deviation of $C_{2}, \sigma_{C 2}$, the normalized standard deviation of $\mathrm{C} 2, \sigma_{C 2 \text {,norm }}$, and the standard deviation in $y(h), \sigma_{y}$. These parameters (in Appendix A) were defined based on the assumption that only common uncertainties occur and systematic errors have been ignored. ${ }^{20}$

The criterion for the selection of the optimum regression range is $\min \left\{\sigma_{C 1}, \sigma_{C 2}, \sigma_{C 1, \text { norm }}, \sigma_{C 2 \text { norm }}, \sigma_{y}\right\}$. Regression coefficients $C_{1}$ and $C_{2}$ obtained for this
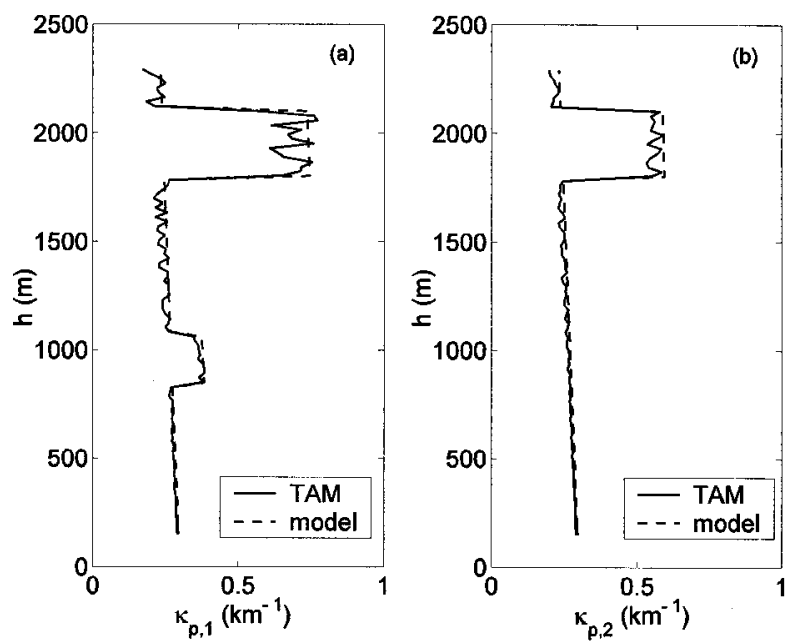

Fig. 4. Vertical profiles of the particulate extinction coefficient obtained with TAM along with the model particulate extinction coefficients: (a) $\kappa_{p, 1}(h)$ at $45^{\circ}$; (b) $\kappa_{p, 2}(h)$ at $90^{\circ}$.
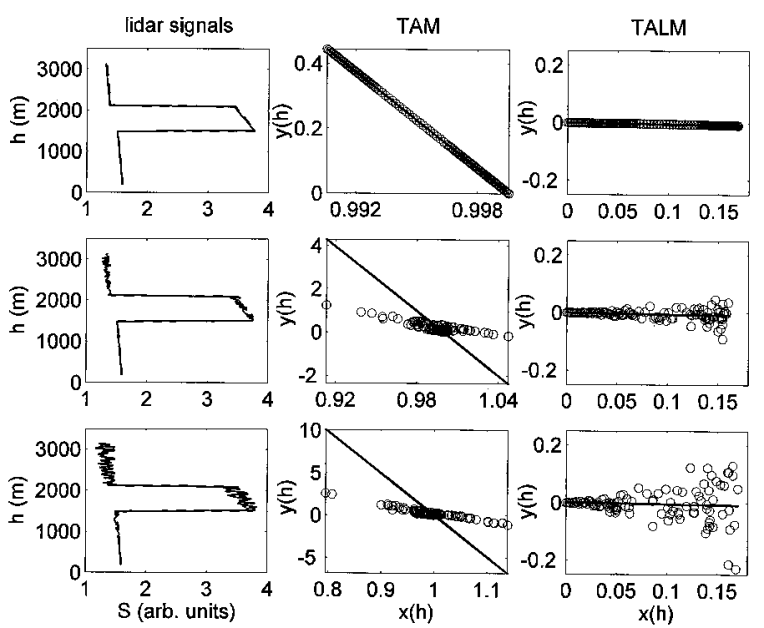

Fig. 5. Comparison between TAM and TALM: left, synthetic lidar signal with an increasing noise level, from top to bottom row (solid line, $\varphi_{1}=75^{\circ}$; dashed line, $\varphi_{2}=90^{\circ}$ ); center, open circles, pairs of $x(h)$ versus $y(h)$ computed with TAM (solid line, the true solution); right, open circles, $x(h)$ versus $y(h)$ computed with TALM (solid line, the true solution).

regression range are used for further data processing. Figure 3 shows the set of pairs $[x(h), y(h)]$ and the best fit, as obtained from the regression analysis, taking into consideration all five parameters described above. Now, when lidar constants $C_{1}$ and $C_{2}$ obtained from the regression analysis are used, the profiles of the weighted extinction coefficients $\kappa_{W, 1}(h)$ and $\kappa_{W, 2}(h)$ can be computed according to Eqs. (4) and (5). Substracting the weighted molecular constituent $a \kappa_{m}(h)$ [see Eq. (8)] yields the final result, the vertical profiles of the particulate extinction coefficient. A direct comparison with the original profile of $\kappa_{p, 1}(h)$ [Fig. 4(a)] and $\kappa_{p, 2}(h)$ [Fig. 4(b)] demonstrates that it is possible with TAM to retrieve the profile of the extinction coefficient fairly accurately even for a not absolutely homogeneous, mildly turbid atmosphere and noise-corrupted lidar data. The profiles are in good agreement with the actual profiles of $\kappa_{p}(h)$.

\section{Two-Angle Logarithmic Method}

The previously introduced straightforward algorithm yields an asymmetric solution for the extinction coefficient. This in turn may significantly affect inversion accuracy, especially in clear atmospheres [see Eq. (12)]. An improved, iterative logarithmic variant of TAM that allows for an averaging procedure and that eliminates this drawback was developed in Ref. 12. This method, the two-angle logarithmic method (TALM), requires horizontal homogeneity in a statistical sense only, i.e., after appropriate averaging. The assumption of statistical homogeneity can be tested reliably with standard statistical methods.

To clarify the difference between TAM and TALM, a brief comparison is presented. Figure 5 shows synthetic lidar signals $S(h)$ in a clear atmosphere at two elevation angles with a cloud layer at altitude. 
Different noise levels are added to the signals. The middle panel shows the resulting $x(h)$ versus $y(h)$ pairs for the three cases as computed with TAM. For the first case (no noise) the regression is stable and the solution is symmetric. However, for increasing noise levels the solution becomes more and more unstable and asymmetric. With TAM no useful information can be extracted from the lidar signal in those cases. Consider the results with TALM (right panel). For the no-noise case the solution is highly accurate, as it was for TAM. But now, even when a large noise constituent is added, the solution remains symmetric; i.e., the $x(h)-y(h)$ pairs fluctuate around the true solution. In those cases the inversion is feasible, and extinction coefficient profiles can be retrieved from the lidar data by using TALM, although the signals were spoiled by high noise levels. Note that the influence of signal noise is equivalent to the influence of small-scale atmospheric inhomogeneity, and hence such atmospheric inhomogeneity results in similar systematic shifts for the TAM inversion, whereas the influence of the same inhomogeneity would be much less for TALM.

\section{Minimization Technique}

Both TAM and TALM make use of a methodology that involves linear regression. However, one can also resort to a method that does not incorporate a regression procedure but rather relies on a minimization technique. This calibration method is developed next. After rearranging and taking the logarithm on both sides of the general TAM equation [Eq. (9)], one obtains

$$
\begin{aligned}
\ln \left[\frac{\kappa_{W, 1}(h)}{\kappa_{W, 2}(h)}\right]= & \ln \left[\frac{S_{1}(h)}{S_{2}(h)}\right]-\ln (A)-\ln [1 \\
& \left.-\frac{2 I_{1}\left(h_{1}, h\right)}{A C_{2}}\right]+\ln \left[1-\frac{2 I_{2}\left(h_{1}, h\right)}{C_{2}}\right],
\end{aligned}
$$

where $A=C_{1} / C_{2}$. The basic TALM assumption used here is that the natural logarithm of the ratio between $\kappa_{W, 1}(h)$ and $\kappa_{W, 2}(h)$ is randomly distributed and is close to zero for all heights $h$. By this definition no systematic heterogeneities occur owing to small-scale atmospheric inhomogeneity, and hence there are also no systematic deviations on the lefthand side of Eq. (16) from zero within the measurement range from $h_{1}$ to $h_{\max }$. To determine constants $A$ and $C_{2}$, one simply has to minimize the right-hand side of Eq. (16). Denoting the left-hand side in Eq. (16) as $\eta(h)$, we can consider Eq. (16) as a minimization function,

$$
\begin{aligned}
\eta(h)= & \ln \left[\frac{S_{1}(h)}{S_{2}(h)}\right]-\ln (A)-\ln \left[1-\frac{2 I_{1}\left(h_{1}, h\right)}{A C_{2}}\right] \\
& +\ln \left[1-\frac{2 I_{2}\left(h_{1}, h\right)}{C_{2}}\right],
\end{aligned}
$$

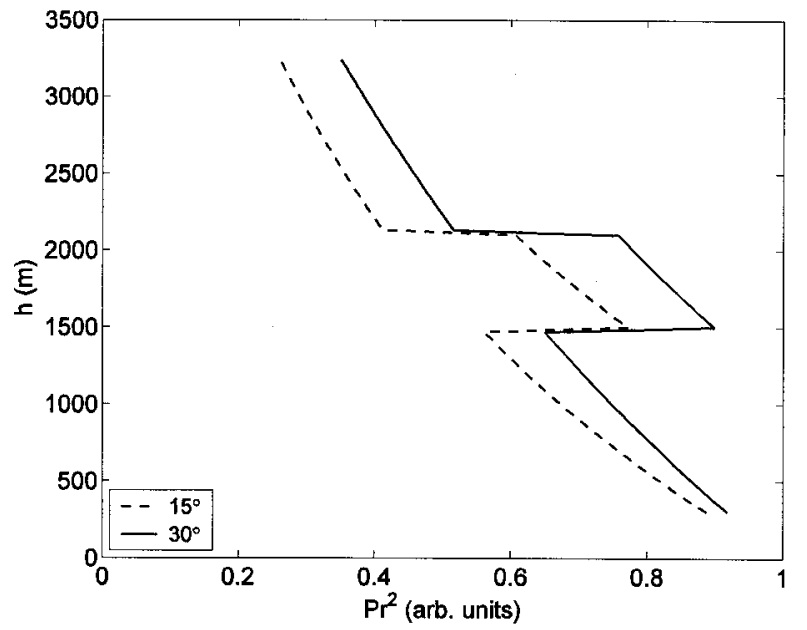

Fig. 6. Synthetic lidar signals at $\varphi_{1}=15^{\circ}$ and $\varphi_{2}=30^{\circ}$ for a homogeneous model atmosphere. The noise-free case is shown.

so that the optimum constants $C_{1}\left(=A C_{2}\right)$ and $C_{2}$ can be found by determining $\min \{\operatorname{abs}[\eta(h)]\}$. Using these constants $C_{1}$ and $C_{2}$, we can then compute the vertical particulate extinction coefficient profile.

To evaluate the performance of the minimization technique, we use synthetic data. The model atmosphere is composed of molecules and particles. Clear atmospheric conditions prevail, but a cloud layer from 1500 to $2100 \mathrm{~m}$ has been included. The noise-free lidar signals are shown in Fig. 6. The minimization function $\eta(h)$ (Fig. 7) yields the lidar solution constants $C_{1}=27.98$ and $C_{2}=28.94$, which are used to compute the profiles of the particulate extinction coefficient. The model profile and the inversion result of $\kappa_{p}(h)$, when the minimization technique is used, agree for both $\varphi_{1}=15^{\circ}$ [Fig. 8(a)] and $\varphi_{2}=30^{\circ}$ [Fig. 8(b)]. The relative error between the model extinction coefficient profile and the extinction coefficient profile retrieved with the minimization technique is $0.0 \%$ for both elevation angles. To test the minimization technique performance more rigor-

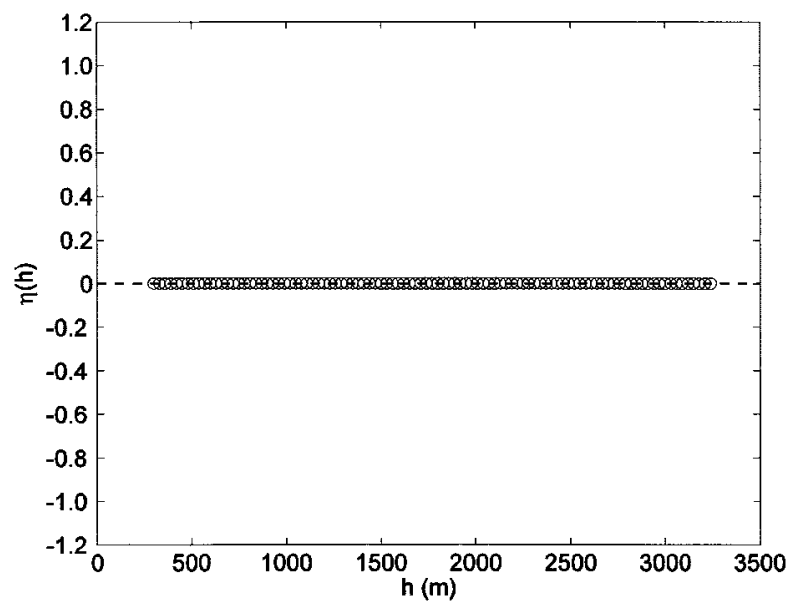

Fig. 7. Minimization function $\eta(h)$ for the homogeneous noisefree case. The $y$ scale has been increased to allow for comparison with the noisy case (Fig. 10). 

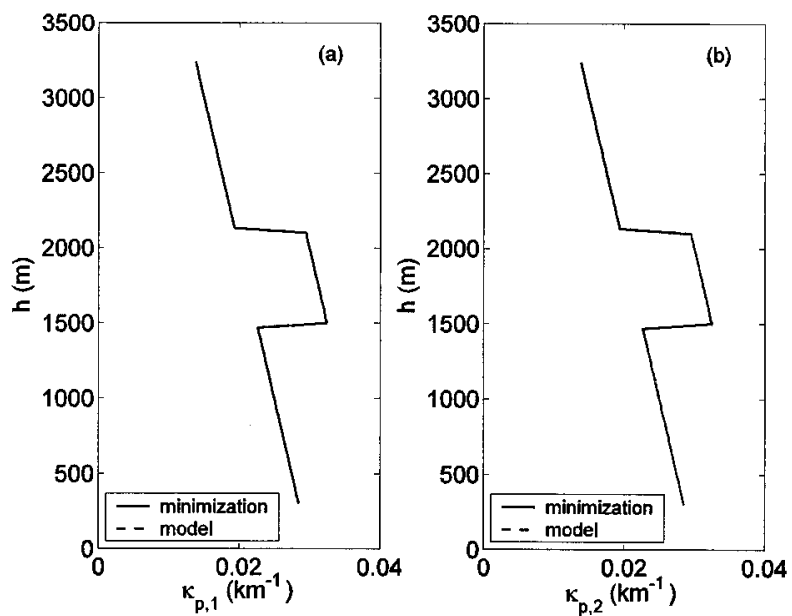

Fig. 8. Model profile of the particulate extinction coefficient and resulting profile by use of the minimization technique for (a) $\varphi_{1}=$ $15^{\circ}$ and (b) $\varphi_{1}=30^{\circ}$. The two lines are indiscernible because the relative error is nil.

ously, we added random noise to the lidar signals (Fig. 9). The mean signal-to-noise ratio SNR is 32 for elevation angle $\varphi_{1}$ and 108 for elevation angle $\varphi_{2}$. Note that the random noise also resembles an inhomogeneous atmosphere. The minimization function $\eta(h)$ shows strong fluctuations due to the noise that has been added, yet it remains symmetric (Fig. 10). The resulting lidar solution constants are $C_{1}=29.20$ and $C_{2}=30.24$. The extinction coefficient profiles for $\varphi_{1}$ and $\varphi_{2}$ are compared with the model profiles in Fig. 11. For this noisy case the relative error between model and minimization results is $17.7 \%$ for $\varphi_{1}$ and $6.4 \%$ for $\varphi_{2}$, which is acceptable considering the large noise constituent in the lidar signals.

We now extend the previous test case by including a local atmospheric inhomogeneity. An additional mildly turbid layer that intersects only with elevation angle $\varphi_{2}=30^{\circ}$ can be seen in the lidar

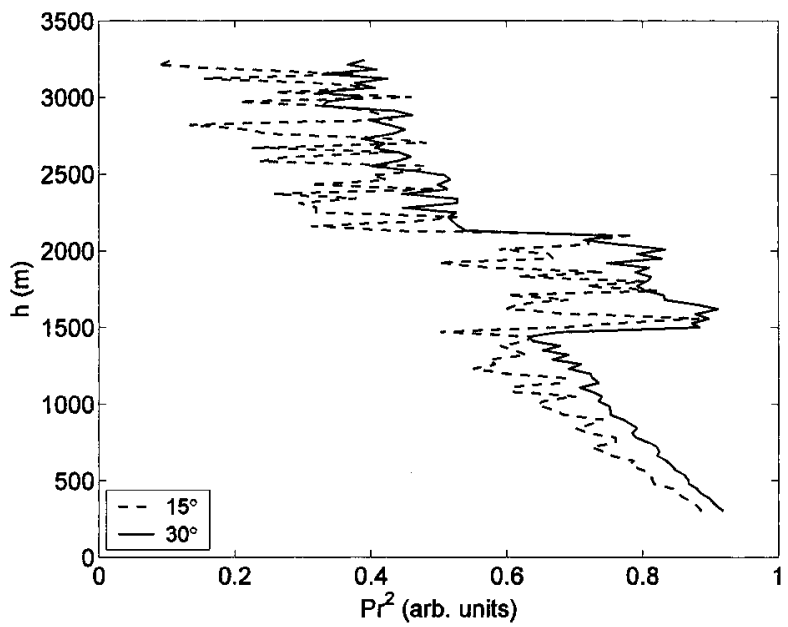

Fig. 9. Synthetic lidar signals at $\varphi_{1}=15^{\circ}$ and $\varphi_{2}=30^{\circ}$ for a homogeneous model atmosphere. The noisy case is shown.

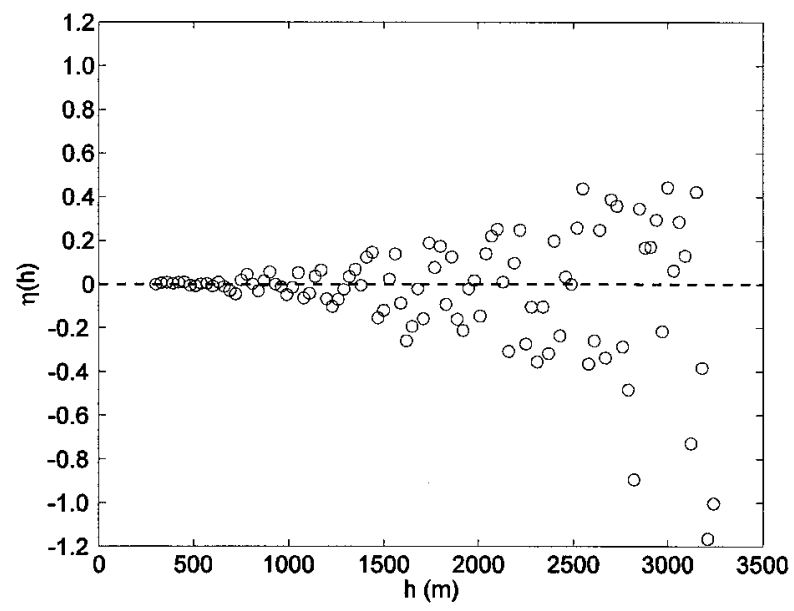

Fig. 10. Minimization function $\eta(h)$ for the homogeneous noisy case.

signal (Fig. 12). First the noise-free case is studied. The minimization function for this inhomogeneous atmosphere is shown in Fig. 13, which yields $C_{1}=27.98$ and $C_{2}=28.94$. Figure 14 shows a comparison between model profiles and results from the minimization technique. For both elevation angles the relative error between the model profile and the inversion result is $3.0 \%$. Next random noise is added to the lidar signals, as shown in Fig. 15. The mean SNR is 54 for $\varphi_{1}=15^{\circ}$ and 172 for $\varphi_{2}=30^{\circ}$. The minimization function (Fig. 16) for this noisy and inhomogeneous case demonstrates that random noise has an effect similar to inhomogeneities, especially in the far field. Strong fluctuations due to noise are predominant, and they even exceed the large value in $\eta(h)$ due to the inhomogeneous layer along $\varphi_{2}$. However, the minimization function remains symmetric. With the resulting solution constants $C_{1}=28.09$ and $C_{2}=$ 29.09 the vertical profiles of the particulate extinction coefficient are computed and presented in Fig.
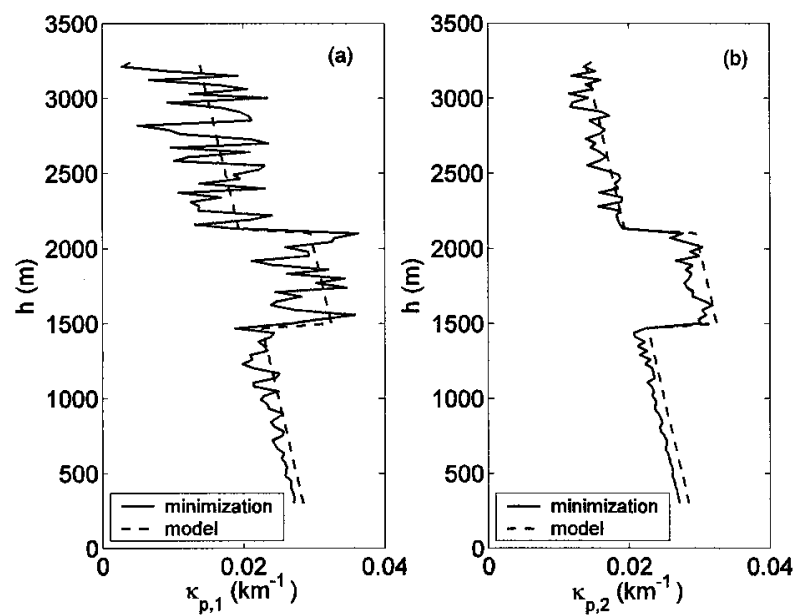

Fig. 11. Model profile of the particulate extinction coefficient and resulting profile when the minimization technique for (a) $\varphi_{1}=15^{\circ}$ and (b) $\varphi_{1}=30^{\circ}$ is used. 


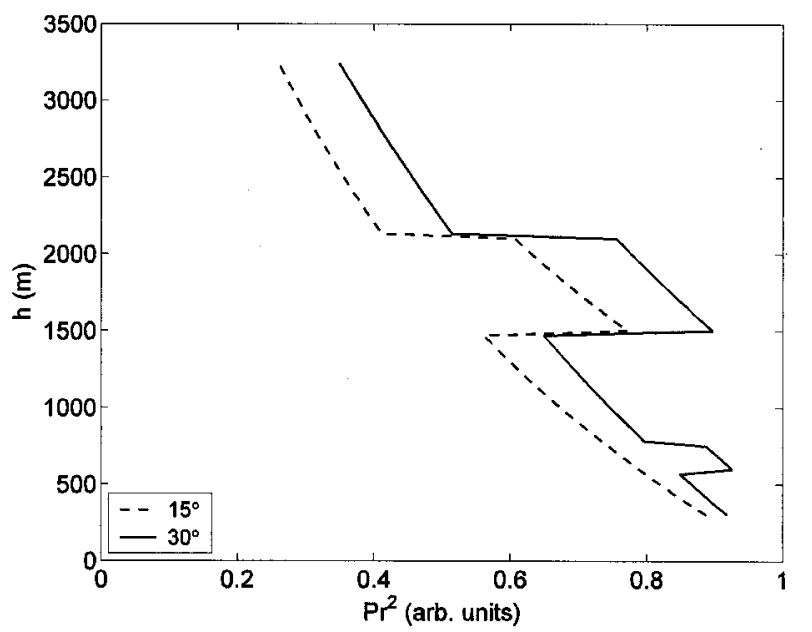

Fig. 12. Synthetic lidar signals at $\varphi_{1}=15^{\circ}$ and $\varphi_{2}=30^{\circ}$ for an inhomogeneous model atmosphere. The noise-free case is shown.

17 together with the model profiles. The relative error between model profiles and inversion results when the minimization technique is used for the noisy and the inhomogeneous case is $10.8 \%$ for $\varphi_{1}$ and $4.0 \%$ for $\varphi_{2}$. Again the relative error remains acceptable, even for the large noise in the lidar signals.

\section{Summary}

Two previously developed methods, a modified twoangle method (TAM) and a logarithmic two-angle method (TALM), for determining the lidar solution constants and subsequently the vertical profiles of the atmospheric extinction coefficient from scanning elastic lidar data have been evaluated and used as a foundation for devising a new approach. The basic form of these methods, TAM, is most applicable in moderately turbid and turbid atmospheres. In clear atmospheres, local inhomogeneities and signal noise

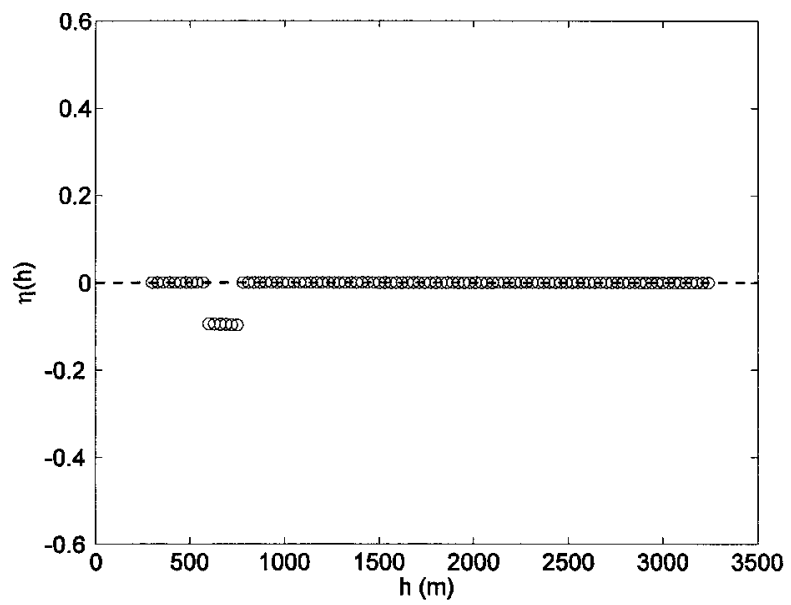

Fig. 13. Minimization function $\eta(h)$ for the inhomogeneous noisefree case. The $y$ scale has been increased to allow for comparison with the noisy case (Fig. 16).
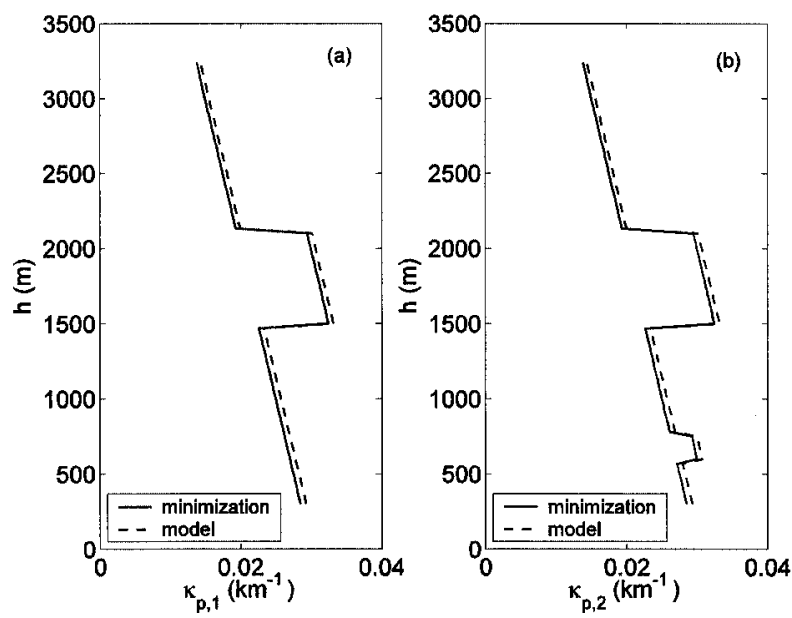

Fig. 14. Model profile of the particulate extinction coefficient and resulting profile when the minimization technique for (a) $\varphi_{1}=15^{\circ}$ and (b) $\varphi_{1}=30^{\circ}$ is used.

can cause a significant systematic offset in the solution constants determined with TAM. This asymmetry is avoided in TALM. Both TAM and TALM allow for averaging and hence increased solution stability. Yet, compared with TAM, the application range of TALM extends to clear atmospheres. Note that these methods differ from conventional multiangle methods.

(1) It is feasible to successfully apply TAM and TALM even if the atmosphere is homogeneous over restricted altitude ranges only, rather than over the entire two-dimensional scan. Sectors where significant horizontal inhomogeneity exists can be excluded from consideration so that only the best available signals are used for the calibration.

(2) The methods do not require strict horizontal homogeneity of the atmosphere under investigation but only horizontal homogeneity in a statistical sense. This statistical homogeneity is achieved by

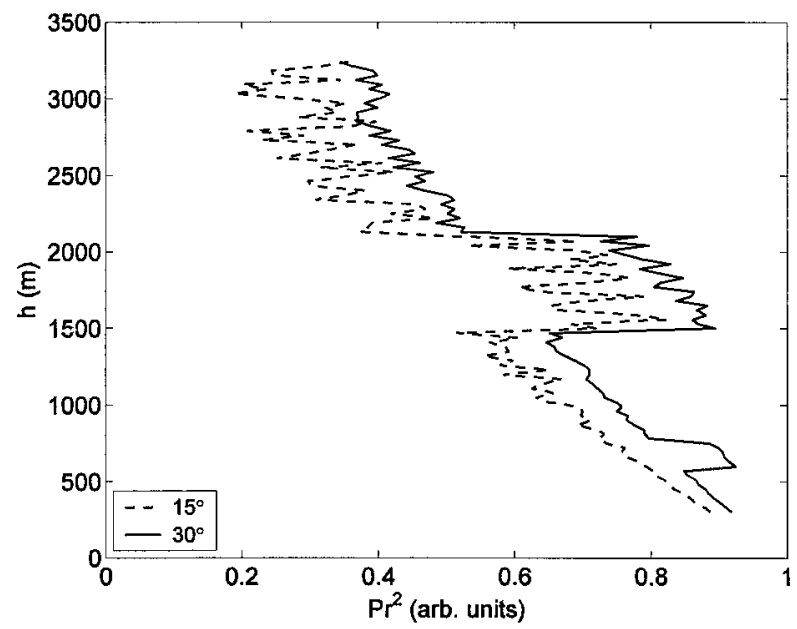

Fig. 15. Synthetic lidar signals at $\varphi_{1}=15^{\circ}$ and $\varphi_{2}=30^{\circ}$ for the inhomogeneous model atmosphere. The noisy case is shown. 


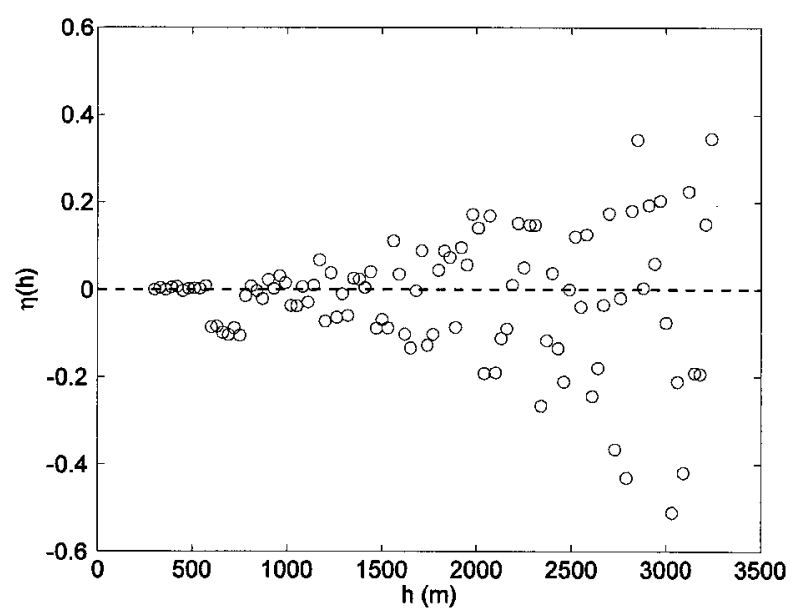

Fig. 16. Minimization function $\eta(h)$ for the inhomogeneous noisy case.

using large sets of two-angle pairs to determine the best solution constants.

(3) TAM and TALM are primarily calibration methods, i.e., methods for determining the solution constants for a particular lidar scan.

Once the constants have been determined, one can retrieve the profiles of atmospheric extinction, investigate possible aerosol (layer) structures, probe cloud layers, or study the transition between the boundary layer and the free atmosphere, not by using the assumption of horizontal homogeneity any longer.

An alternative to the regression procedure in TALM is a newly developed method that is based on TALM but uses a minimization technique that replaces the regression procedure. The method has been tested extensively with synthetic data. It provides a means of obtaining the lidar solution constants for scanning lidar data, which in turn can be used to determine the vertical profile of the particulate extinction coefficient. As for TAM and TALM, measurements along at least two elevation angles are
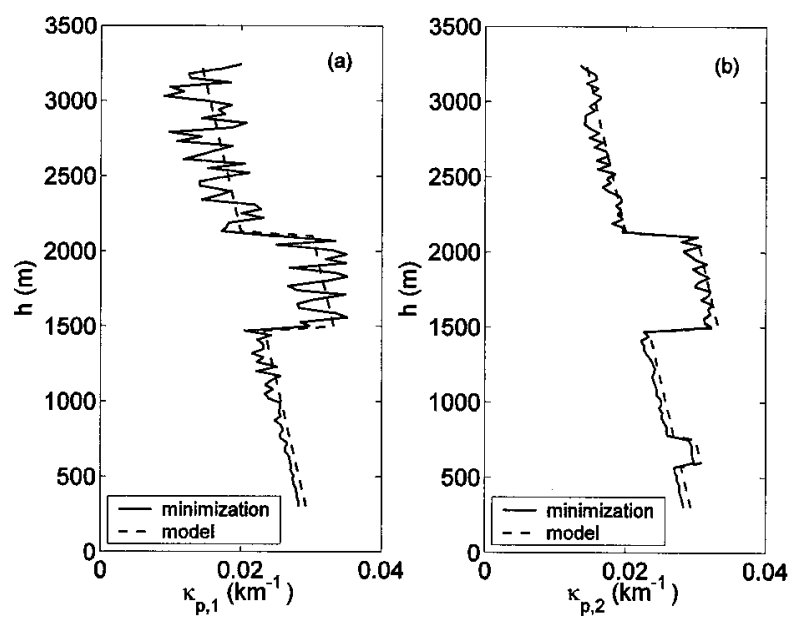

Fig. 17. Model profile of the particulate extinction coefficient and the resulting profile when the minimization technique for (a) $\varphi_{1}=$ $15^{\circ}$ and (b) $\varphi_{1}=30^{\circ}$ is used. required. The solution is stable, even if excessive noise is being added to the lidar signal. It also proved to be stable when a model atmosphere with local areas of inhomogeneity has been investigated.

\section{Appendix A: Parameters for Determining the Optimum Range for Regression and the Regression Coefficients $C_{1}$ and $C_{2}$}

Standard deviation of $C_{1}$ :

$$
\sigma_{C_{1}}=\left(\frac{\sigma_{y}^{2}}{\Delta^{\prime}} \sum_{i} x_{i}^{2}\right)^{1 / 2} .
$$

Normalized standard deviation of $C_{1}$ :

$$
\sigma_{C_{1, \mathrm{norm}}}=\left|\sigma_{C_{1}} / C_{1}\right| \text {. }
$$

Standard deviation of $C_{2}$ :

$$
\sigma_{C_{2}}=\left(N \frac{\sigma_{y}^{2}}{\Delta^{\prime}}\right)^{1 / 2}
$$

Normalized standard deviation of $C_{2}$ :

$$
\sigma_{C_{2, \text { norm }}}=\left|\sigma_{C_{2}} / C_{2}\right| \text {. }
$$

Standard deviation of $y(h)$ :

$$
\sigma_{y}=\left[\frac{1}{N-2} \sum_{i}\left(y_{i}-C_{1}-C_{2} x_{2}\right)^{2}\right]^{1 / 2},
$$

where

$$
\Delta^{\prime}=N \sum_{i} x_{i}{ }^{2}-\left(\sum_{i} x_{i}\right)^{2} .
$$

\section{References}

1. M. Kano, "On the determination of backscattered and extinction coefficient of the atmosphere by using a laser radar," Pap. Meteorol. Geophys. 19, 121-129 (1968).

2. P. M. Hamilton, "Lidar measurement of backscatter and attenuation of atmospheric aerosol," Atmos. Environ. 3, 221-223 (1969).

3. J. D. Spinhirne, J. A. Reagan, and B. M. Herman, "Vertical distribution of aerosol extinction cross section and inference of aerosol imaginary index in the troposphere by lidar technique," J. Appl. Meteorol. 19, 426-438 (1980).

4. V. A. Kovalev and V. M. Ignatenko, "Method for determining atmospheric transparency," USSR Patent, SU 1163217 A, USSR Bulletin 23, 3521598/24-25 (1985).

5. J. D. Spinhirne, S. Chudamani, J. F. Cavanaugh, and J. L. Bufton, "Aerosol and cloud backscatter at 1.06, 1.54, and 0.53 $\mu \mathrm{m}$ by airborne hard-target-calibrated Nd:YAG/methane Raman lidar," Appl. Opt. 36, 3475-3490 (1997).

6. Y. Sasano and H. Nakane, "Quantitative analysis of RHI lidar data by an iterative adjustment of the boundary condition term in the lidar solution," Appl. Opt. 26, 615-616 (1987).

7. T. Takamura, Y. Sasano, and T. Hayasaka, "Tropospheric aerosol optical properties derived from lidar, sunphotometer, and optical particle counter measurements," Appl. Opt. 33, 7132-7140 (1994).

8. Y. Sasano, "Tropospheric aerosol extinction coefficient profiles derived from scanning lidar measurements over Tsukuba, Japan from 1990 to 1993," Appl. Opt. 35, 49414952 (1996). 
9. D. Gutkowicz-Krusin, "Multiangle lidar performance in the presence of horizontal inhomogeneities in atmospheric extinction and scattering," Appl. Opt. 32, 3266-3272 (1993).

10. M. Sicard, P. Chazette, J. Pelon, J. G. Won, and S. C. Yoon, "Variational method for the retrieval of the optical thickness and the backscatter coefficient from multiangle lidar profiles," Appl. Opt. 41, 493-502 (2002).

11. V. M. Ignatenko, "Data processing method for the slope lidar paths," in Proceedings of the Main Geophysical Observatory, G. P. Gushchin, ed. (Main Geophysical Observatory, Leningrad, Russia, 1991), No. 533, pp. 76-78.

12. V. A. Kovalev, M. Pahlow, and M. B. Parlange, "Elimination of asymmetry in the two-angle lidar-equation solution for aerosol extinction profiles," in Lidar Remote Sensing in Atmospheric and Earth Sciences, Proceedings ILRC21, L. Bissonnette, G. Roy, and G. Vallée, eds. (Defence Research and Development Canada (DRDC) Valcartier, Val-Bélair, Canada, 2002), pp. 621-624.

13. M. Pahlow, "Atmospheric boundary layer dynamics and inversion technologies to obtain extinction coefficient profiles in the atmosphere from elastic lidar," Ph.D. dissertation (Johns Hopkins University, Baltimore, Md., 2002).
14. V. A. Kovalev, "Stable near-end solution of the lidar equation for clear atmospheres," Appl. Opt. 42, 585-591 (2003).

15. E. V. Browell, S. Ismail, and S. T. Shipley, "Ultraviolet DIAL measurements of $\mathrm{O}_{3}$ profiles in regions of spatially inhomogeneous aerosols," Appl. Opt. 24, 2827-2836 (1985).

16. T. Takamura and Y. Sasano, "Ratio of aerosol backscatter to extinction coefficients as determined from angular scattering measurements for use in atmospheric lidar applications," Opt. Quantum Electron. 19, 293-302 (1987).

17. B. T. N. Evans, "Sensitivity of the backscatter/extinction ratio to changes in aerosol properties: implications for lidar," Appl. Opt. 27, 3299-3306 (1988).

18. T. L. Anderson, S. J. Masonis, D. S. Covert, R. J. Charlson, and M. J. Rood, "In situ measurements of the aerosol extinctionto-backscatter ratio at a polluted continental site," J. Geophys. Res. 105, 26,907-26,915 (2000).

19. I. Mattis, A. Ansmann, D. Müller, U. Wandinger, and D. Althausen, "Dual-wavelength Raman lidar observations of the extinction-to-backscatter ratio of Saharan dust," Geophys. Res. Lett. 29, 10.1029/2002GL014721 (2002).

20. P. R. Bevington and D. K. Robinson, Data Reduction and Error Analysis for the Physical Sciences (McGraw-Hill, Boston, 1992). 